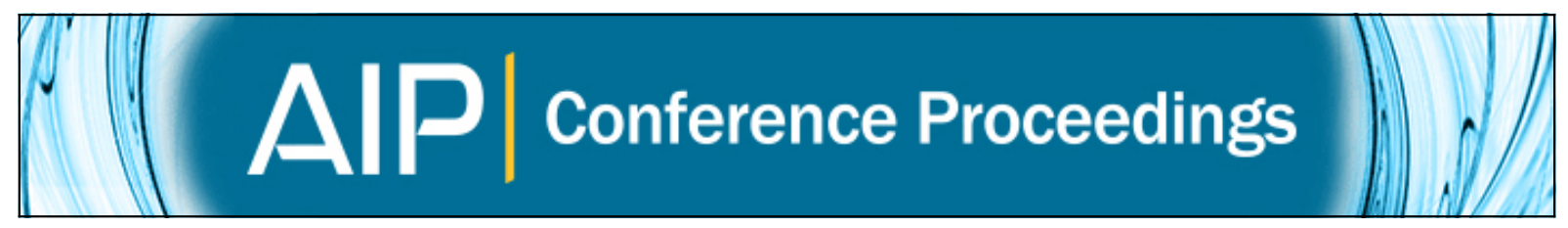

\title{
Linear and quadratic sufficiency and commutativity
}

Sandra S. Ferreira, Dário Ferreira, and Célia Nunes

Citation: AIP Conference Proceedings 1479, 1694 (2012); doi: 10.1063/1.4756496

View online: http://dx.doi.org/10.1063/1.4756496

View Table of Contents: http://scitation.aip.org/content/aip/proceeding/aipcp/1479?ver=pdfcov

Published by the AIP Publishing

\section{Articles you may be interested in}

Comparison of linear discriminant analysis and logistic regression for data classification

AIP Conf. Proc. 1522, 1159 (2013); 10.1063/1.4801262

Noise propagation in linear and nonlinear inverse scattering

J. Acoust. Soc. Am. 121, 2743 (2007); 10.1121/1.2713671

Commuting quantum traces for quadratic algebras

J. Math. Phys. 46, 083516 (2005); 10.1063/1.2007587

Magnetically linear and quadratic hexanitrocuprates

J. Appl. Phys. 50, 1825 (1979); 10.1063/1.327183

Properties of "Quadratic" Canonical Commutation Relation Representations

J. Math. Phys. 10, 1661 (1969); 10.1063/1.1665013 


\title{
Linear and Quadratic Sufficiency and Commutativity
}

\author{
Sandra S. Ferreira, Dário Ferreira, Célia Nunes \\ Mathematics Department and Center of Mathematics, University of Beira Interior \\ 6200 Covilhã, Portugal
}

\begin{abstract}
Given a mixed model let $T$ be the orthogonal projection matrix on the range space spanned by the mean vector. If the model has variance- covariance matrix $\sigma^{2} V$ we use commutative Jordan algebras to show that $T y$ is both linear sufficient and linear complete and that $T y, y^{\prime} V^{+} y$ with $V^{+}$the Moore-Penrose inverse of $V$ is quadratic sufficient whenever $T$ and $V$ commute.
\end{abstract}

Keywords: Linear sufficiency, quadratic sufficiency, orthogonal projection, linear completeness

AMS: $62 \mathrm{H} 15,62 \mathrm{~J} 10$

\section{INTRODUCTION}

Zmyślony (1978) showed that when the orthogonal projection matrix, OPM, $T$, on the range space $\Omega$ spanned by the mean vector $\mu=X \beta$ commutes with the variance-covariance matrix $V$, the least square estimators, LSE, of linear estimable functions $c^{\prime} \beta$ [or of estimable vectors] are best linear unbiased estimators, BLUE.

We now consider linear and quadratic sufficiency for models in which $T$ and $V$ commute. Following Mueller (1987), we say that:

- $L y$ is linearly sufficient if for every linear estimable function $c^{\prime} \beta$ the BLUE is given by $b^{\prime} L Y$;

- $\left(L Y ; y^{\prime} U y\right)$ is quadratically sufficient for the model $Y \sim Q\left(X \beta, \sigma^{2} V\right) ; \beta \in R^{p}, \sigma^{2}>0$, if $L y$ is linearly sufficiency and there exists a symmetric matrix $A$ and a real $\alpha$ such that $y^{\prime} L A L y+\alpha y^{\prime} U y$ is a best quadratic unbiased estimator, BQUE, for $f \sigma^{2}$, with $f$ known.

In the last expression, $Y \sim Q\left(X \beta, \sigma^{2} V\right)$ indicates $Y$ to be quasi-normal, this is itt's first four order moments are related in the same way as for normal vectors, with $\mu=X \beta$ and variance-covariance matrix $\sigma^{2} V$. It is easy to see that the linear sufficiency of $L Y$ does not depend on the introduction of $\sigma^{2}$ in the definitions of the variance covariance matrix of $Y$. Besides these notions of sufficiency we will also consider, following again Mueller (1987), the linear completeness. Thus $L y$ is linearly complete if any linear function $c^{\prime} \beta$ with null mean value for every $\beta \in R^{p}$ is almost surely null. In our study of quadratic completeness we will use commutative Jordan algebras, which we will discuss in the next section. Then we consider linear sufficiency and completeness and finally quadratic sufficiency.

\section{COMMUTATIVE JORDAN ALGEBRAS}

We restrict ourselves to commutative Jordan algebras, CJA, of symmetric matrices. There will be linear spaces constituted by symmetric matrices that commute and containing the squares of this matrices.

Seely (1971) showed that each CJA $\mathscr{A}$ has an unique basis constituted by pairwise orthogonal OPM, POOPM, the principal basis, $p b(\mathscr{A})$ of $\mathscr{A}$. According to Schott (1997), the matrices of a family $M=\left\{M_{1}, \ldots, M_{w}\right\}$ of symmetric matrices commute if and only if they are diagonalized by an orthogonal matrix $P$. Then we will have $M \subset \mathscr{V}(P)$ with $\mathscr{V}(P)$ the family of symmetric matrices diagonalized by $P$ which is a CJA. Since intersection of CJA gives CJA (ref?), the intersection of all CJA that contain $M$ will be a CJA, the CJA $\mathscr{A}(M)$ generated by $\mathscr{A}$.

With $Q=\left\{Q_{1}, \ldots, Q_{m}\right\}=p b(\mathscr{A})$, given $M \in \mathscr{A}$ we will have $M=\sum_{j=1}^{m} a_{j} Q_{j}$ it being easy to see that the MOORE- 
PENROSE inverse of $M$ is

$$
M^{+}=\sum_{j=1}^{m} a_{j}^{+} Q_{j}
$$

putting $a_{j}^{+}=a_{j}^{-1} \quad[0]$ when $a_{j} \neq 0 \quad[=0], j=1, \ldots, m$. Thus any CJA contains the MOORE-PENROSE inverse of its matrices.

If $Q \in \mathscr{A}$ is an OPM it will be idempotent then $Q=\sum_{j=1}^{m} q_{j} Q_{j}$ with the $q_{1}, \ldots, q_{m}$ equal to 0 or 1 , since the $Q_{1}, \ldots, Q_{m}$ are pairwise orthogonal and idempotent.

Now

$$
Q M Q=\sum_{j=1}^{m} q_{j} a_{j} q_{j} Q_{j}=\sum_{j \in C} a_{j} Q_{j}
$$

with $C=\left\{j: q_{j} a_{j} q_{j} \neq 0\right\}$, and so

$$
(Q M Q)^{+}=\sum_{j \in C} a_{j}^{-1} Q_{j}=Q M^{+} Q
$$

since we also have $C=\left\{j: q_{j} a_{j}^{+} q_{j} \neq 0\right\}$.

\section{LINEAR SUFFICIENCY AND COMPLETENESS}

Let $N(W)$ be the nullity space of matrix $W$, then, according to Mueller (1987), $L y$ is linearly sufficient for the model with mean vector $X \beta$ and variance covariance matrix $V$ if and only if $x_{1} \cap x_{2} \subset \triangle_{1}$, with

$$
\left\{\begin{array}{l}
x_{1}=N(L) \\
x_{2}=\Omega \oplus \triangle_{1} \\
\triangle_{1}=V N\left(X^{\prime}\right)
\end{array}\right.
$$

where $\oplus$ indicates direct sum of subspaces and

$$
V N\left(X^{\prime}\right)=\left\{V u ; u \in N\left(X^{\prime}\right)\right\}
$$

It is well known that, $N\left(X^{\prime}\right)=R(X)^{\perp}$ where $\perp$ indicates orthogonal complement, so we also have

$$
\triangle_{1}=V R(X)^{\perp}=\{V z ; z \perp R(X)\} .
$$

Moreover, see again Mueller (1987), Ly is linearly complete if and only if

$$
x_{1} \cap x_{2}=\triangle_{1}
$$

We now establish

Lemma 1 We have $\triangle_{1}=R\left(V T^{c}\right)$, with $T^{c}=I_{n}-T$.

Proof: $\quad$ The thesis follows from $V N\left(X^{\prime}\right)=V R(X)^{\perp}=V R\left(T^{c}\right)=R\left(V T^{c}\right)$ since $R(X)^{\perp}=R(T)^{\perp}=R\left(T^{c}\right)$.

Corollary 1 When $V$ and $T$ commute, $x_{2}=\Omega \boxplus \triangle_{1}$, where $\boxplus$ stands for orthogonal direct sum of subspaces.

Proof: Since $V$ and $T\left[V\right.$ and $\left.T^{c}\right]$ commute, $\triangle_{1}=R\left(V T^{c}\right)=R\left(T^{c} V\right) \subset R\left(T^{c}\right)=\Omega^{\perp}$, thus $\Omega=R(T)$ and $\triangle_{1}$ will be orthogonal.

Proposition 1 When $V$ and $T$ commute, $T y$ will be linearly sufficient and linearly complete. 
Proof: We must have $x_{1}=N(T)=\Omega^{\perp}$ thus, according to Corollary 3.1 of Lemma $1 x_{1} \cap x_{2}=\triangle_{1}$, which establishes the thesis.

Besides this, see Scheffé (1959), $c^{\prime} \beta$ is estimable when and only when $c=X^{\prime} u$, so that $c^{\prime} \beta=u^{\prime} X \beta$. Now the LSE of $c^{\prime} \beta$ is $\widetilde{c^{\prime} \beta}=u^{\prime} X \widetilde{\beta}$, with $\widetilde{\beta}=\left(X^{\prime} X\right)^{+} X^{\prime} Y$. Since $T=X\left(X^{\prime} X\right)^{+} X^{\prime}$ we will have $c^{\prime} \beta=u^{\prime} T y$ which emphasizes the linear sufficiency of $T y$. In this way, we relate the linear sufficiency of $T y$ with the Zmyślony (1978) result on LSE. Now if $V$ depends on a vector $\sigma^{2}$ of variance components, so that

$$
V=V\left(\sigma^{2}\right), \sigma^{2} \in \ominus
$$

For $V$ commuting with $T$ we must have

$$
T V\left(\sigma^{2}\right)=V\left(\sigma^{2}\right) T ; \quad \sigma^{2} \in \ominus
$$

For instance given the mixed model

$$
y=\sum_{i=0}^{w} X_{i} \beta_{i}
$$

with $\beta_{0}$ fixed and $\beta_{1}, \ldots, \beta_{w}$ independent, with null mean vectors and variance covariance matrices $\sigma_{1}^{2} I_{c_{1}}, \ldots, \sigma_{w}^{2} I_{c_{w}}, y$ will have mean vectors and variance covariance matrices given by

$$
\left\{\begin{array}{l}
\mu=X_{0} \beta_{0} \\
V=\sum_{i=1}^{w} \sigma_{i}^{2} V_{i} ; \quad \theta \in \Theta=\mathscr{X}_{i=1}^{w}[0 ;+\infty[
\end{array}\right.
$$

with $\mathscr{X}$ indicating cartesian product and $V_{i}=X_{i} X_{i}^{\prime}, i=1, \ldots, w$, while the orthogonal projection matrix on the space spanned by $\mu$ will now be written as

$$
T=X_{0}\left(X_{0}^{\prime} X_{0}\right)^{+} X_{0}^{\prime}
$$

For $V$ and $T$ to commute it is necessary and sufficient that

$$
T V_{i}=V_{i} T, i=1, \ldots, w
$$

This conditions holds namely for models with commutative orthogonal block structure, COBS, see Fonseca et al (2007). Then in these models $T y$ will be linearly sufficient and linearly complete.

\section{QUADRATIC SUFFICIENCY}

Given the model

$$
Y \sim Q\left(X \beta, \sigma^{2} V\right)
$$

(Ly, $\left.y^{\prime} U y\right)$ enjoys quadratic sufficiency if and only if, see Mueller (1987),

$$
x_{1} \cap x_{2}=\triangle_{1} \cap \triangle_{2} \cap \triangle_{3},
$$

with $x_{1}, x_{2}$ and $\triangle_{1}$ defined as above and

$$
\left\{\begin{array}{l}
\triangle_{2}=N\left(X^{\prime} U\right) \\
\triangle_{3}=N\left(I_{n}-\alpha V U\right)
\end{array}\right.
$$

The equality between subspaces is assumed to hold for the $\alpha$ that is considered in the definition of quadratic sufficiency. When $T$ and $V$ commute, taking $T^{c}=I_{n}-T$, considering $T^{c}$ the complement subspace of matrix $T$, the matrices of a family $M=\left\{T, T^{c}, V\right\}$ commute and generate a CJA $\mathscr{A}=\mathscr{A}(M)$ that contains $V^{+}, Q=V V^{+}, Q_{1}=T Q$ and $Q_{2}=T^{c} V$. Matrices $Q, Q_{1}$ and $Q_{2}$ are OPM and $Q_{1}$ and $Q_{2}$ are pairwise orthogonal.

We now establish 
Lemma 2 When $T$ and $V$ commute and we take $L=T$ and $U=V^{+}$, we have

$$
\left\{\begin{array}{l}
x_{1}=N(T)=\Omega^{\perp} \\
x_{2}=\Omega \boxplus \nabla_{2} \\
\triangle_{1}=\nabla_{2} \\
\triangle_{2}=\nabla_{1}^{\perp}
\end{array}\right.
$$

and

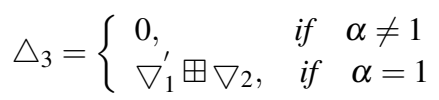

with $\nabla_{1}=R\left(Q^{\prime}\right)$ and $\nabla_{2}=R\left(Q_{2}\right)$.

Proof: We already saw that taking $L=T$ we have $x_{1}=\Omega^{\perp}$ and that, according to Lemma $\mathbf{1}, \triangle_{1}=R\left(V T^{c}\right)$. Since $R(V)=R(Q)$ we also will have

$$
\triangle_{1}=R\left(V T^{c}\right)=R\left(T^{c} V\right)=T^{c} R(V)=T^{c} R(Q)=R\left(T^{c} Q\right)=R\left(Q_{2}\right)=\nabla_{2},
$$

thus $\Omega$ and $\triangle_{1}=\nabla_{2}$ are orthogonal so $x_{2}=\Omega \boxplus \nabla_{2}$. Next we have

$$
\begin{aligned}
& \triangle_{2}=N\left(X^{\prime} V^{+}\right)=R\left(V^{+} X\right)^{\perp}=\left(V^{+} R(X)\right)^{\perp}=\left(V^{+} R(T)\right)^{\perp}=R\left(V^{+} T\right)^{\perp}= \\
& =R\left(T V^{+}\right)^{\perp}=\left(T R\left(V^{+}\right)\right)^{\perp}=(T R(Q))^{\perp}=(R(T Q))^{\perp}=R\left(Q_{1}\right)^{\perp}=\nabla_{1}^{\perp} .
\end{aligned}
$$

Lastly we point out that, with $Q^{c}=I_{n}-Q$ we have

$$
I_{n}-\alpha V V^{+}=I_{n}-\alpha Q=Q^{c}+(1-\alpha) Q
$$

which is invertible when $\alpha \neq 1$. When $\alpha=1$, we have $I_{n}-Q=Q^{c}$ and $N\left(I_{n}-Q\right)=R(Q)=Q^{c}=\nabla_{1} \boxplus \nabla_{2}$, which completes the proof. $\square$

We now may establish the

Proposition 2 When $T$ and $V$ commute, taking $\alpha=1, \quad\left(T y, y^{\prime} V^{+} y\right)$ is quadratic complete for the model $Y \sim$ $Q\left(X \beta, \sigma^{2} V\right)$.

Proof: The thesis follows from, according to Lemma 2

$$
\left\{\begin{array}{l}
x_{1} \cap x_{2}=\nabla_{2} \\
\triangle_{1} \cap \triangle_{2} \cap \triangle_{3}=\nabla_{2}
\end{array}\right.
$$

since $\nabla_{2}$ being a subspace of $\nabla_{1}^{\perp}$ and of $\left(\nabla_{1} \boxplus \nabla_{2}\right)$.

\section{ACKNOWLEDGEMENTS}

The authors are grateful to the anonymous referees for their useful comments and remarks. This work was partially supported by the center of Mathematics, University of Beira Interior, under the project PEst-OE/MAT/UI0212/2011.

\section{REFERENCES}

1. M. Fonseca; J. M. Mexia and R. Zmyślony. Jordan algebras, generating pivot variables and orthogonal normal models. J. Interdiscip. Math., 10, 2, 305Ü-326, 2007.

2. J. Mueller.Sufficiency and completeness in the linear model. Journal of Multivariate Analysis, 21 (2), 312-323, 1987.

3. J. Seely. Quadratic subspaces and completeness. Ann. Math. Statis. 42, 2, 710-721, 1971

4. H. Scheffé. The Analysis of Variance. John Willey \& Sons. New York, 1959.

5. J. R. Schott. Matrix Analysis for Statistics. Wiley Series in Probability and Statistics. New York, 1996.

6. R. Zmyślony. A characterization of best linear unbiased estimators in the general linear model. Mathematical Statistics and Probability Theory, Proc. Sixth Internat. Conf., Wisla/Pol. 1978, Lect. Notes Stat., 2, Springer, New York-Berlin, 365-373, 1980. 